in vivo $32: 1419-1426(2018)$

doi:10.21873/invivo.11394

\title{
Evaluation of Porcine Hybrid Bone Block for Bone Grafting in Dentistry
}

\author{
SE EUN KIM*, EUNSEOK LEE*, KWANGSIK JANG, KYUNG MI SHIM and SEONG SOO KANG \\ College of Veterinary Medicine, BK21 Plus Project Team and Biomaterial R\&BD Center, \\ Chonnam National University, Gwangju, Republic of Korea
}

\begin{abstract}
Background/Aim: The purpose of this study was to develop hybrid bone blocks using porcine-derived collagen and low crystalline porcine-derived hydroxyapatite to overcome the disadvantages of commonly used bone grafts in dentistry. Materials and Methods: Collagen was added to hydroxyapatite particles to increase the spatial integration of particulate bone grafts. Physicochemical examination and in vivo tests were performed to analyze scaffold's characteristics and evaluate bone regeneration. Results: Porcine hybrid bone block had an irregular and interconnecting macroporous structure that was adequate for bone regeneration and bone ingrowth, and showed a good space-occupying ability to become well positioned. In addition, it showed higher angiogenesis and biodegradability than Bio-Oss Collagen ${ }^{\circledR}$, a commercialized bone graft used in dental clinics. Conclusion: Our results suggest that improved collagen hybrid bone block can be generated when porcine cancellous bone particles and collagen were reasonably mixed. This hybrid bone block was easy in handling had flexibility, good biodegradability and provided bone regeneration.
\end{abstract}

Bone graft materials are necessary to fill large bone defects in dentistry. Among the various types of bone grafts, the

This article is freely accessible online.

*These Authors contributed equally to this work.

Correspondence to: Seong Soo Kang, DVM, Ph.D., College of Veterinary Medicine, Chonnam National University, 77, Yongbongro, Buk-gu, Gwangju, 61186, Republic of Korea. Tel: +82 625302877, Fax: +82 625302809, e-mail: vetkang@chonnam.ac.kr and Kyung Mi Shim, DVM, PhD, College of Veterinary Medicine, Chonnam National University, 77, Yongbong-ro, Buk-gu, Gwangju, 61186, Republic of Korea. Tel: +82 625302880, Fax: +82 625302809, e-mail: simchung-98@hanmail.net

Key Words: Porcine hybrid bone block, collagen, hydroxyapatite, biodegradability, bone regeneration. particle type is commonly used because it enables dense packing in an intraosseous defect with irregular forms (1). However, particulate bone grafts are susceptible to external compressive force and are not easy to be well localized within the defect. Hence, a barrier membrane is required to maintain the grafted particulate bone within the defect and prevent collapse of the site. Because the use of a barrier membrane increases costs and operation time, bone substitutes that can be adjusted in the bone defect without a membrane should be developed (2).

A combination of organic components such as collagen and inorganic particles is a promising strategy for ideal bone grafting because collagen and hydroxyapatite are the main components of bone (3). Collagen is the most abundant protein in mammals, and provides structural and mechanical support to tissues with good biodegradability and biocompatibility (4). Collagen has been widely used as a scaffold in medical applications and tissue engineering, such as for skin and vascular grafts, hemostats, adhesives, and drug delivery (5). Despite its excellent biological properties, collagen has poor mechanical strength and must be crosslinked by physical or chemical treatments, or combined with other polymers or inorganic materials.

Bio-Oss Collagen ${ }^{\circledR}$ (BC) shows enhanced handling characteristics and provides good hemostatic properties by mixing $90 \%$ Geistlich Bio-Oss ${ }^{\circledR}$ granules with $10 \%$ porcine collagen. Several preclinical and clinical studies have shown that Bio-Oss Collagen ${ }^{\circledR}$ exhibits favorable osteoconductivity and space-making properties. However, Bio-Oss ${ }^{\circledR}$ granules tend to be absorbed very slowly and remain in the new bone tissue. Moreover, the use of bovine-derived materials such as Bio-Oss Collagen ${ }^{\circledR}$ poses a risk of bovine spongiform encephalopathy (BSE).

The aim of the present study was to develop porcine hybrid bone block (PHBB) with characteristics such as easy handling, flexibility, good biodegradability and free from BSE. To confirm the clinical efficacy of the PHBB, we compared bone regeneration and biodegradability of $\mathrm{PHBB}$ with those of BC. 


\section{Materials and Methods}

Preparation of porcine hybrid bone block. Type I collagen was extracted from porcine skin. Briefly, the porcine dermis homogenized with pepsin and $0.5 \mathrm{M}$ acetic acid was incubated for $24 \mathrm{~h}$ at $4^{\circ} \mathrm{C}$. The insoluble parts were removed and salted out with $5 \mathrm{M} \mathrm{NaCl}$. Pellets were obtained by centrifugation and dialyzed against distilled water for $24 \mathrm{~h}$. The pure collagen was freeze dried for 3 days.

Porcine cancellous bone was trimmed and cut into pieces. Cancellous bones were defatted with toluene and deproteinized with ethylenediamine. The resultants were subjected to heat treatment at $350^{\circ} \mathrm{C}$.

The extracted porcine type I collagen was dissolved in $0.05 \mathrm{M}$ acetic acid and adjusted to $\mathrm{pH} 5 \pm 0.5$ with $1 \mathrm{M} \mathrm{NaOH}$. The collagen solution was cross-linked with 1-ethyl-3-(3-dimethylaminopropyl) carbodiimide and its core agent N-hydroxysuccinimide (both SigmaAldrich, St. Louis, MO, USA) and then dialyzed against distilled water for $24 \mathrm{~h}$.

The cross-linked collagen solution was mixed to porcine cancellous bone particles $(250-850 \mu \mathrm{m})$ at a ratio of $1: 9(\mathrm{w} / \mathrm{w})$ and freeze-dried for 2 days (PHBB).

Both BC and PHBB were prepared in the shape of a disc of diameter $8 \mathrm{~mm}$, weighing $25 \mathrm{mg}$. The discs were sterilized with ethylene oxide gas and aerated for animal studies.

Characterization of $P H B B$. BC and PHBB were imaged by lowvoltage scanning electron microscopy (LV-SEM, JEOL, Tokyo, Japan) to compare their surface morphology.

Chemical characterization of PHBB was carried out by obtaining infrared spectra of $\mathrm{BC}, \mathrm{PHBB}$, porcine cancellous bone, and type I collagen using ATR-FTIR spectroscopy (Bruker TENSOR 37, Bruker AXS. Inc., Germany). The transmission and ATR spectra of each sample were recorded from 4,000 and $600 \mathrm{~cm}^{-1}$ with 64 scans at a resolution of $4 \mathrm{~cm}^{-1}$.

The evaluation of mechanical properties of PHBB was performed at room temperature with a texture analyzer (TMS-Pro, Food Technology Corporation, Sterling, VA, USA). BC and PHBB samples $(4 \times 6 \times 7 \mathrm{~mm})$ were immersed in PBS for hydration for $1 \mathrm{~h}$, and then loaded in uniaxial compression at a displacement rate of $10 \mathrm{~mm} / \mathrm{min}$ to achieve compression of $\sim 50 \%$ of the original sample height. Moreover, flexibility of the PHBB was evaluated by checking resilience of the wet sample after the removal of compression.

Animals. Eight-week-old male Sprague-Dawley rats (weighing 250$280 \mathrm{~g}$, SamTako, Osan, Korea) were used in this study. Animals were housed in polycarbonate cages and kept under standard conditions $\left(23 \pm 2^{\circ} \mathrm{C}, 50 \pm 10 \%\right.$ humidity, $12 / 12$ light-dark cycle, with access to water and food ad libitum). The animal care and surgical protocol were approved by the Institutional Animal Care and Use Committee of Chonnam National University and carried out in accordance with the Guidelines for Animal Experiments of Chonnam National University (CNU IACUC-YB-R-2016-42).

Animal surgery. Animals were anesthetized using $10 \mathrm{mg} / \mathrm{kg}$ xylazine (Rompun, Bayer, Leverkusen, Germany) and $40 \mathrm{mg} / \mathrm{kg}$ ketamine (Ketamine, Yuhan Co., Seoul, Korea) by intraperitoneal injection. The parietal region of rats was shaved and disinfected with $10 \%$ povidone-iodine and $70 \% \mathrm{EtOH}$, and then the cranium was exposed by incision of the skin and periosteum. An $8-\mathrm{mm}$ critical sized calvarial defect was created using a trephine bur, and then $\mathrm{BC}$ or PHBB was grafted into the defect site. The periosteum was closed using 4-0 absorbable sutures (Surgisorb ${ }^{\circledR}$, Samyang, Seoul, Korea), and then 3-0 non-absorbable sutures (Dafilon ${ }^{\circledR}$, B.Braun, Melsungen, Germany) were used to close the skin. Five animals in each group were sacrificed by $\mathrm{CO}_{2}$ at 4 and 8 weeks after operation.

Micro-CT analysis. After harvesting the cranium of rats, microcomputed tomography (CT) scanning was conducted using the Skyscan 1172 Desktop X-ray microtomograph (Bruker, Billerica, MA, USA) at $50 \mathrm{kVp}$ and $200 \mu \mathrm{A}$ to evaluate bone mineral density and bone volume of the scaffolds before and after implantation.

Histological analysis. Specimens were fixed with $10 \%$ buffered formalin and decalcified with Calci-Clear ${ }^{\mathrm{TM}}$ Rapid (National Diagnostics, Atlanta, GA, USA). Samples were then dehydrated in an ascending series of alcohol and embedded in paraplast (Sherwood Medical Industries, Lane Cove, Australia). Embedded specimens were cut to 5 - $\mu \mathrm{m}$ sections with a microtome (Cambridge Instrument, Somerville, MA, USA). Each slide was stained with hematoxylin and eosin, Masson's trichrome, and Goldner's trichrome and observed under a microscope.

Statistical analysis. The experimental data were managed and calculated by using a statistical software (SPSS 21.0, SPSS Inc., Chicago, IL, USA) to compare different groups. Mann-Whitney $U$ tests were used for statistical analysis.

\section{Results}

Analysis of surface morphology. The surfaces of BC and PHBB were scanned by low-voltage scanning electron microscopy (LV-SEM) (Figure 1). LV-SEM images showed that both $\mathrm{BC}$ and $\mathrm{PHBB}$ have structures with irregular and interconnecting macroporosity. Both $\mathrm{BC}$ and $\mathrm{PHBB}$ had microstructures in which collagen fibers were embedded with bovine or porcine bone particles.

ATR-FTIR spectroscopy. Chemical characteristics of BC, PHBB, porcine cancellous bone, and type I collagen were evaluated by using ATR-FTIR spectroscopy. Porcine cancellous bone exhibited distinctive peaks at frequencies of $1,454,1,444,1,020$, and $600-550 \mathrm{~cm}^{-1}$. Porcine type I collagen showed five characteristic absorption bands at the frequencies of $3,296,2,920,1,647,1,544$, and $1,236 \mathrm{~cm}^{-1}$ (Figure 2). In case of $\mathrm{BC}$, two absorption band peaks were observed at 1020 and $600-550 \mathrm{~cm}^{-1}$. PHBB exhibited additional peaks between 1,714 and $1,433 \mathrm{~cm}^{-1}$ as well as distinctive peaks at 1,020 , and $600-550 \mathrm{~cm}^{-1}$.

Mechanical properties. Uniaxial external forces were applied to evaluate the compressive strength of dry/wet $\mathrm{BC}$ and PHBB. Both BC and PHBB showed significant differences in mechanical properties between dry and wet samples at room temperature. The dry compressive strength of the $\mathrm{BC}$ 

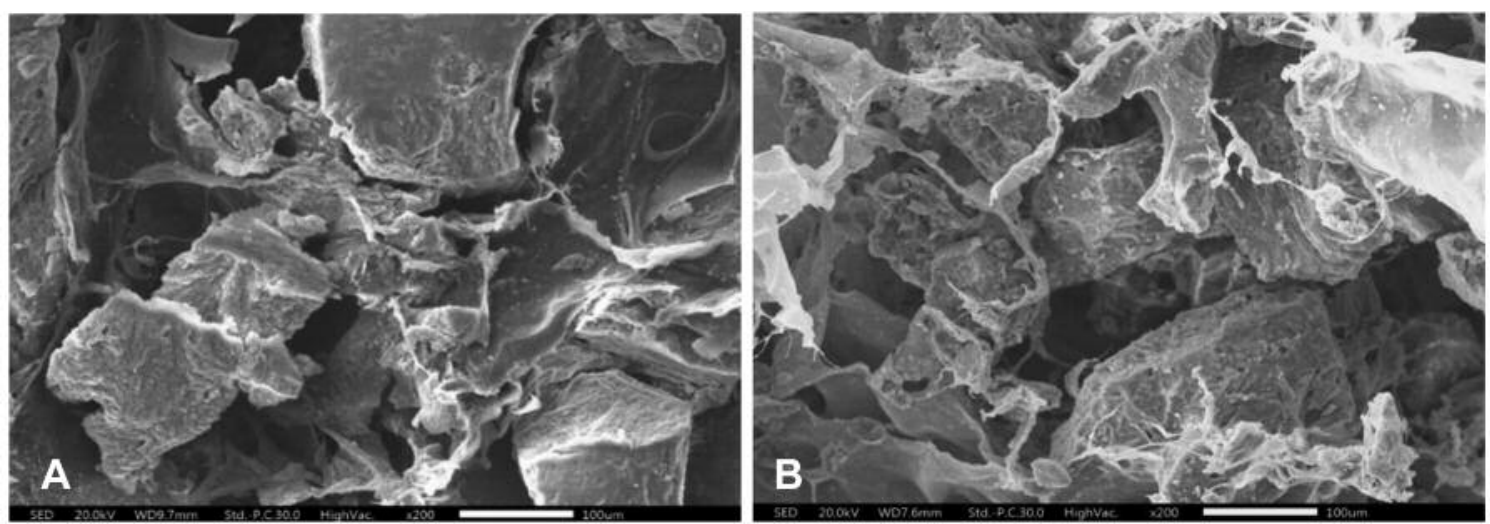

Figure 1. LV-SEM images of bone block surface. (A) Bio-Oss Collagen ${ }^{\circledR}(B C)(\times 200),(B)$ porcine hybrid bone block (PHBB) (x200). Cross-linked collagen and macroporous structures of the surface were observed in both bone grafts.

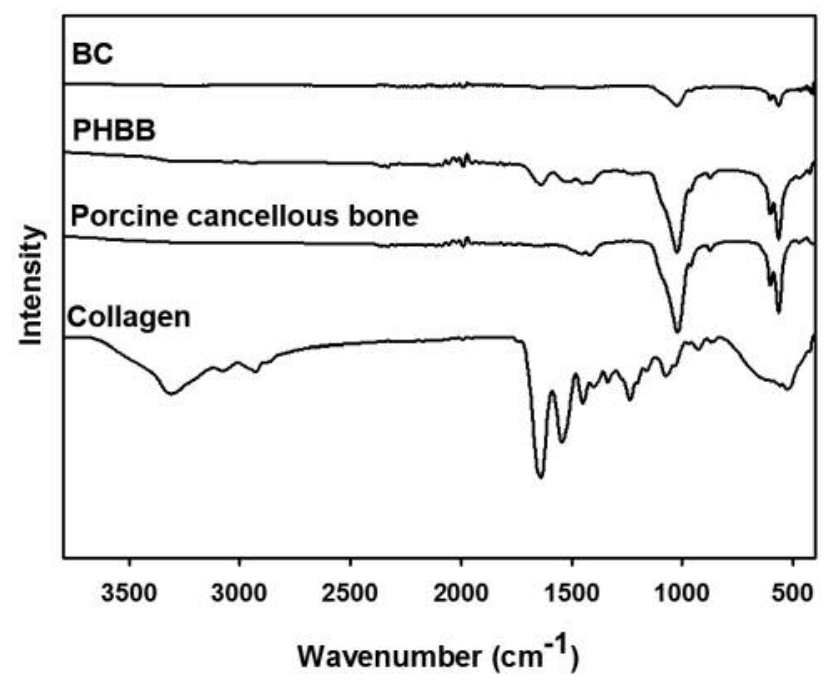

Figure 2. ATR-FTIR spectra of BC, PHBB, porcine cancellous bone, and collagen.

was $53.49 \pm 11.59 \mathrm{~N}$, whereas the wet strength was $4.86 \pm 0.88$ $\mathrm{N}$. In comparison, the PHBB exhibited dry compressive strength of $11.22 \pm 1.53 \mathrm{~N}$ and wet compressive strength of $2.33 \pm 0.37 \mathrm{~N}$ (Figure 3). The resilience of wet PHBB after the removal of compression is shown in Figure 4.

Micro-CT analysis. Both BC and PHBB were well positioned within the calvarial defects (Figure 5). New bone formation was greater in $\mathrm{BC}$ and $\mathrm{PHBB}$ groups than in the critical defect (CD) group.

In micro-CT analysis, bone mineral density (BMD) and bone volume (BV) were increased over time in all groups (Figure 6A and B). Both BMD and BV were significantly

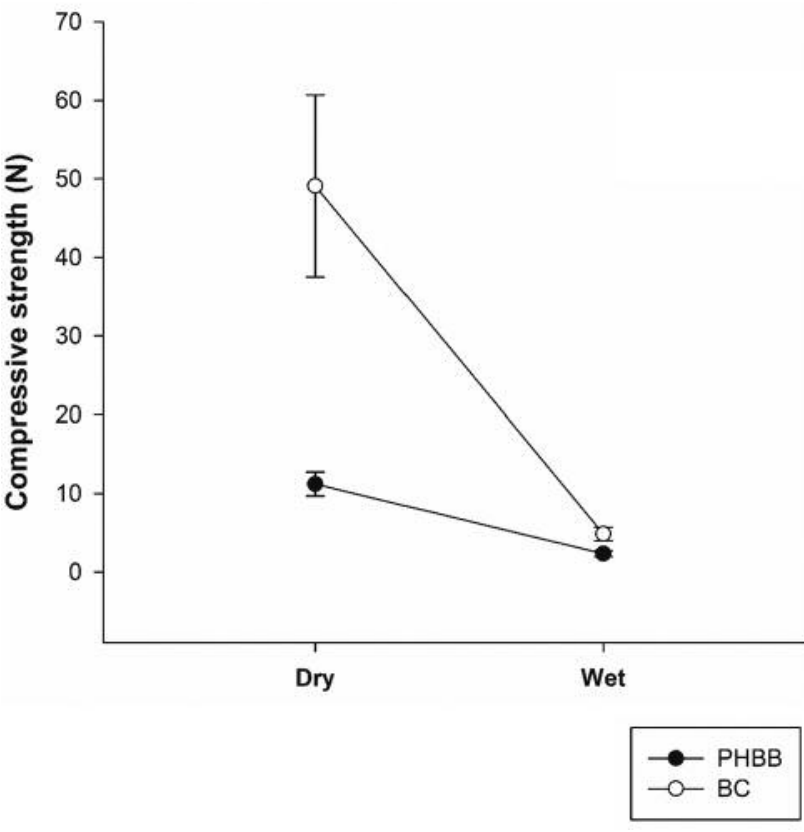

Figure 3. Compressive strength of $P H B B$ and BC. Data are expressed as means $\pm \operatorname{SEM}$ ( $n=5$ per group).

higher in defects containing BC or PHBB than untreated defects at 4 and 8 weeks $(p<0.01)$. In addition, BMD and $\mathrm{BV}$ of the BC group were higher than those of the PHBB group $(p<0.05)$. However, the BC-PHBB ratio of BMD and $\mathrm{BV}$ were decreased at 8 weeks after implantation from 1.91 to 1.23 (BMD) and from 2.00 to $1.22(\mathrm{BV})$ compared to those of scaffolds before implantation.

Histological findings. In both $\mathrm{BC}$ and $\mathrm{PHBB}$ groups, the bone graft space was maintained well without collapse unlike 

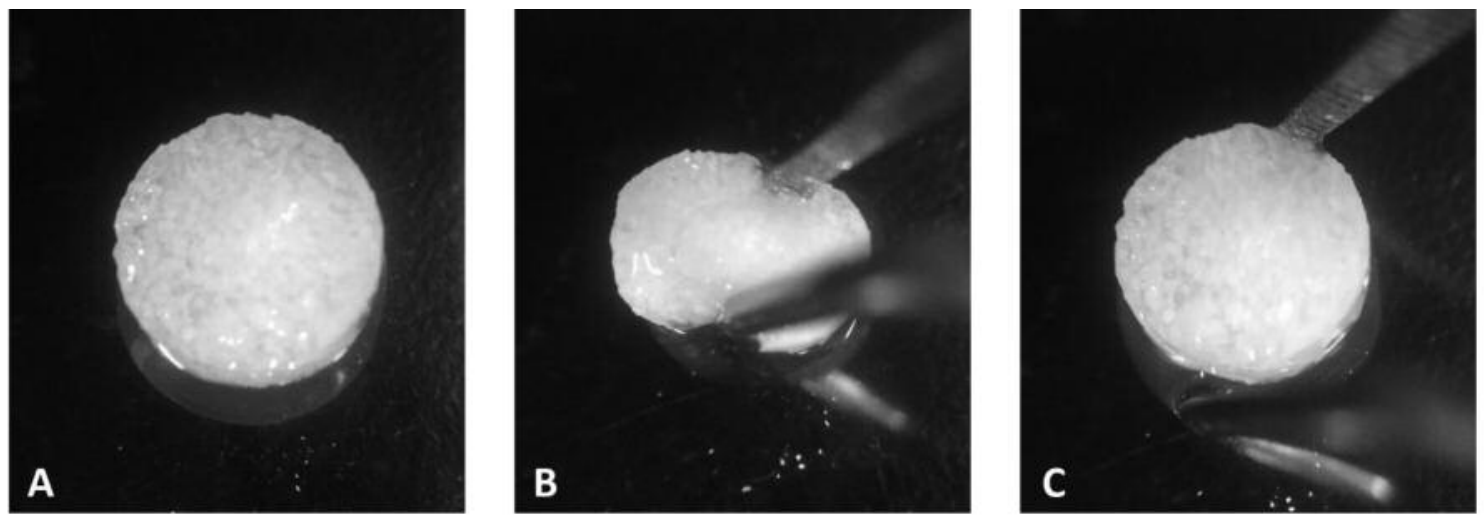

Figure 4. Macroscopic images of PHBB. (A) Before compression, (B) during compression, and (C) after compression.
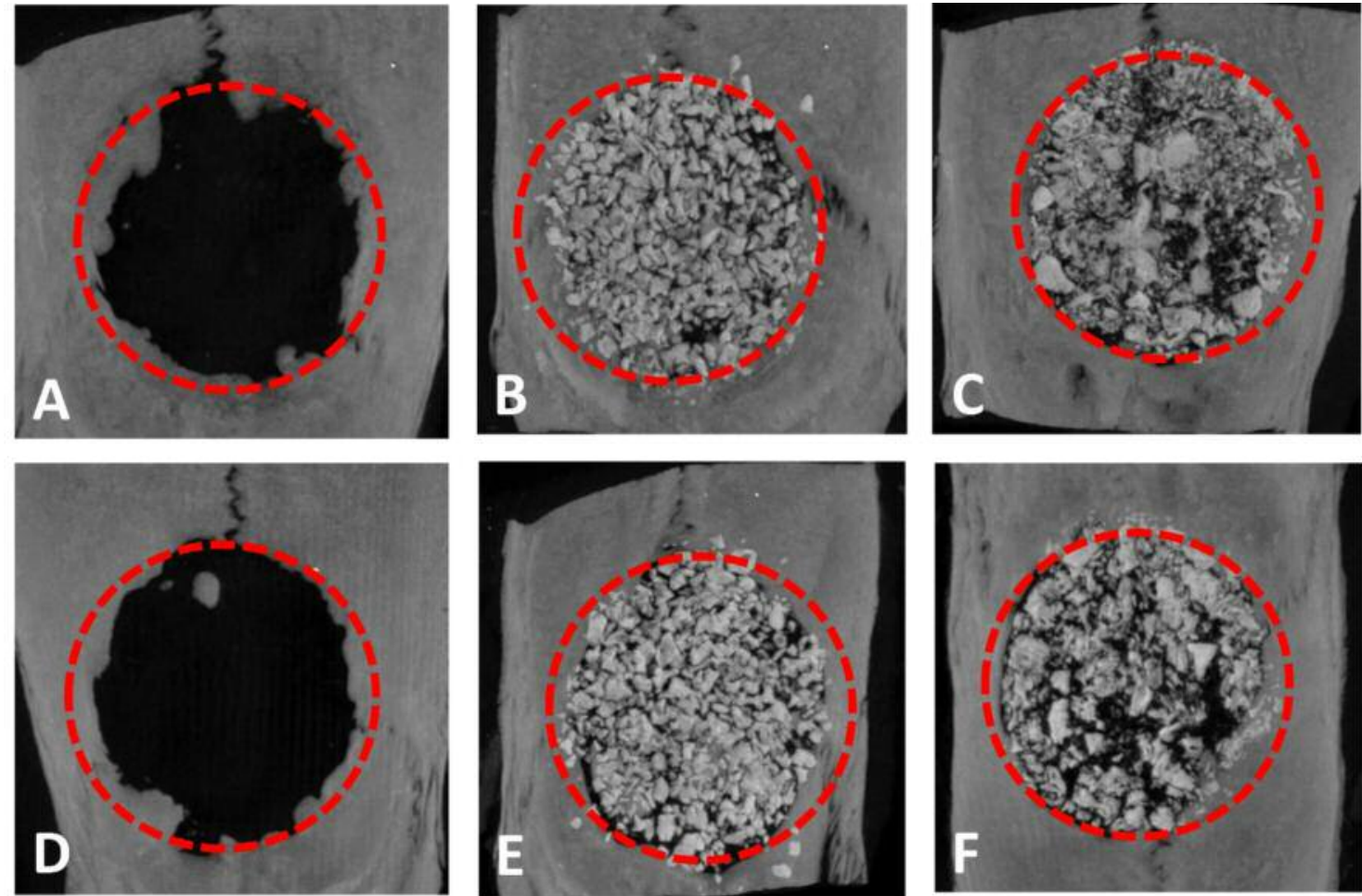

Figure 5. Micro CT 3D images of rat calvarial defects at 4 and 8 weeks after implantation. (A) The CD group at 4 weeks, (B) the BC group at 4 weeks, (C) the PHBB group at 4 weeks, $(D)$ the CD group at 8 weeks, $(E)$ the BC group at 8 weeks, and $(F)$ the PHBB group at 8 weeks.

in the CD group (Figure 7A, E and I). At week 4, bone-like material and new bone formation were observed along the surface of the graft materials in both BC and PHBB groups. However, the PHBB group showed higher angiogenesis and less residual grafts than the BC group (Figure 7).

At week 8, the defect site was mostly filled with fibrous connective tissue in the CD group (Figure 8). Relatively, the defect sites of BC and PHBB groups were filled with residual grafts, bone-like material, and newly formed bone in addition to fibrous connective tissue (Figure 8E and I). Moreover, new bone formation above the dura mater appeared characteristically in both BC and PHBB groups, which was not observed at 4 weeks after bone grafting. The newly formed bone tended to become more mature in both 
A

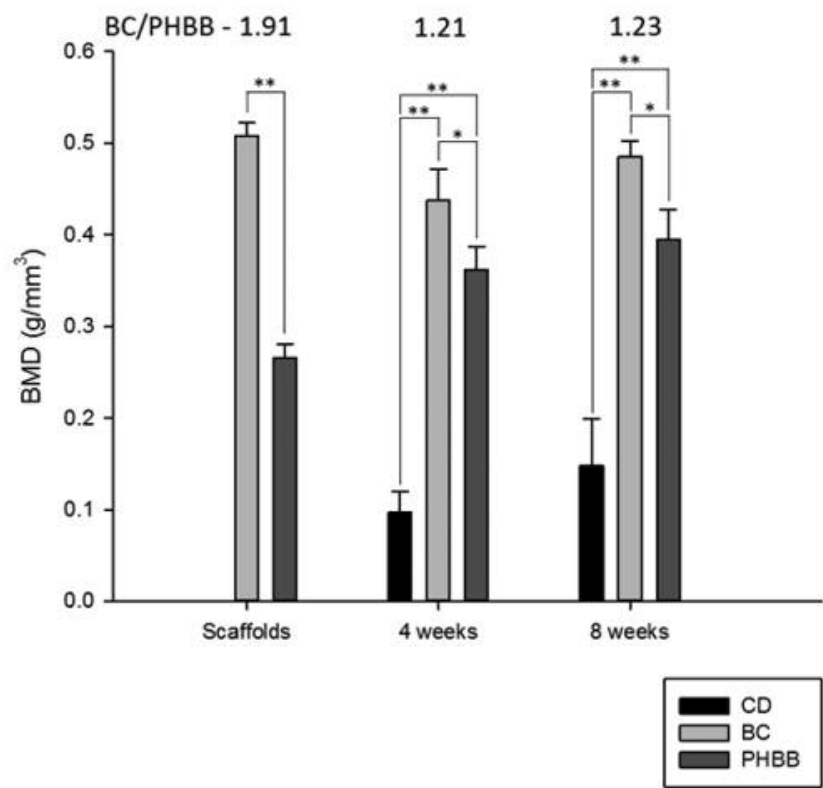

B

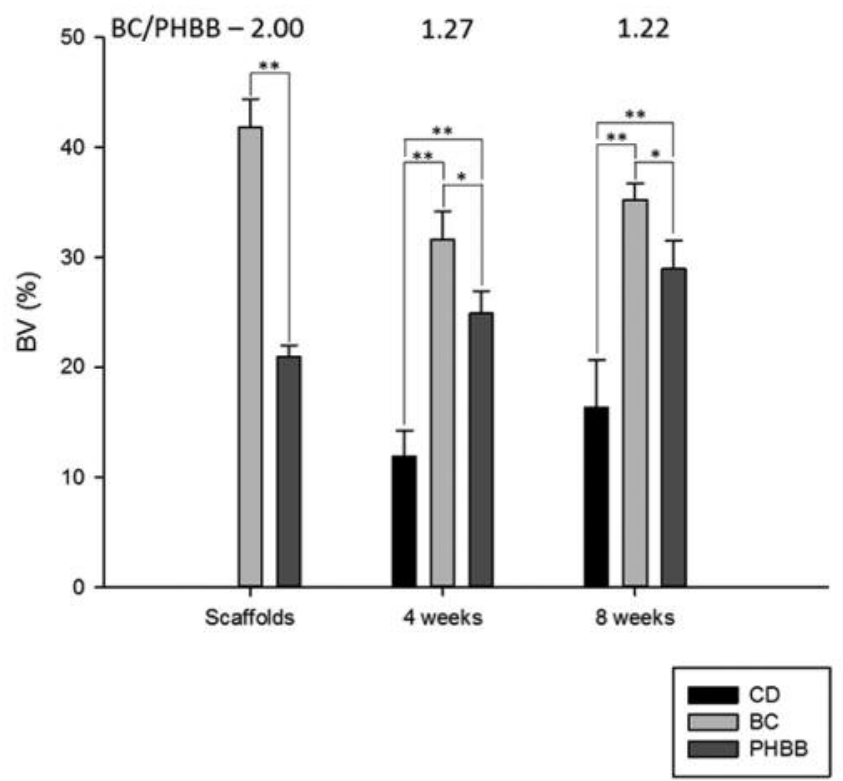

Figure 6. Micro CT measurement of bone formation in rat calvarial defects after implantation. Bone mineral density (A) and bone volume (B) of raw and implanted scaffolds in the defect sites. ${ }^{*} p<0.05, * * p<0.01$. Data are expressed as means \pm SEM ( $n=5$ per group).

experimental groups compared to that at week 4. In the PHBB group, replacement of the grafted materials with bony tissue was prominent.

\section{Discussion}

Bone grafts play a pivotal role in filling extensive bone defects in dentistry. Particulate bone grafts are favored because they fill sufficiently irregular intraosseous defects during dental treatment and maxillofacial surgery (1). However, particulate bone grafts may escape from the grafted site, and thus additional materials and methods such as a barrier membrane are needed to place the grafted materials in the proper position and avoid collapse of the grafted site. The use of a barrier membrane involves additional expenses and prolonged surgical time, exposing patients to greater discomfort. Thus, bone substitutes with a good space maintenance capacity without any additional materials are necessary for better clinical outcomes (2).

Bone consists of many components, among which collagen and hydroxyapatite are the most abundant in the organic and inorganic fractions, respectively $(3,6)$. Thus, many researchers have developed bone substitutes mimicking the properties of live bone tissue by using collagen and hydroxyapatite. Particularly, collagen is one of the most promising materials for the development of scaffolds for clinical use and tissue engineering because it shows high biocompatibility, high biodegradability, and low antigenicity. However, collagen is vulnerable to external force because of its poor mechanical strength, and so the use of collagen as a biomaterial is limited. Thus, material reinforcement such as chemical or physical crosslinking must be carried out for application of collagen in biomaterials science (7).

Bio-Oss Collagen ${ }^{\circledR}$ (BC) is a widely used bone graft that consists of bovine cancellous bone particles and porcine collagen fibers. Despite the many advantages of BC such as enhanced handling characteristics and good hemostatic properties, it shows low biodegradability and may bear the risk of prion transmission (8). The fear of BSE is a particularly important limitation of bovine-derived bone substitutes because of the long latency of prions from 1 to over 50 years. In a previous study, we developed low crystalline hydroxyapatite from porcine cancellous bone by low heat treatment to overcome the weakness of bovinederived bone grafts, and confirmed the improved biodegradation and bone regeneration capacities of this material (9).

In this study, we added porcine-derived type I collagen to porcine-derived low crystalline hydroxyapatite to achieve properties such as easy handling, flexibility, good biodegradability and elimination of the risk for BSE. To evaluate the biodegradability and bone regeneration of 

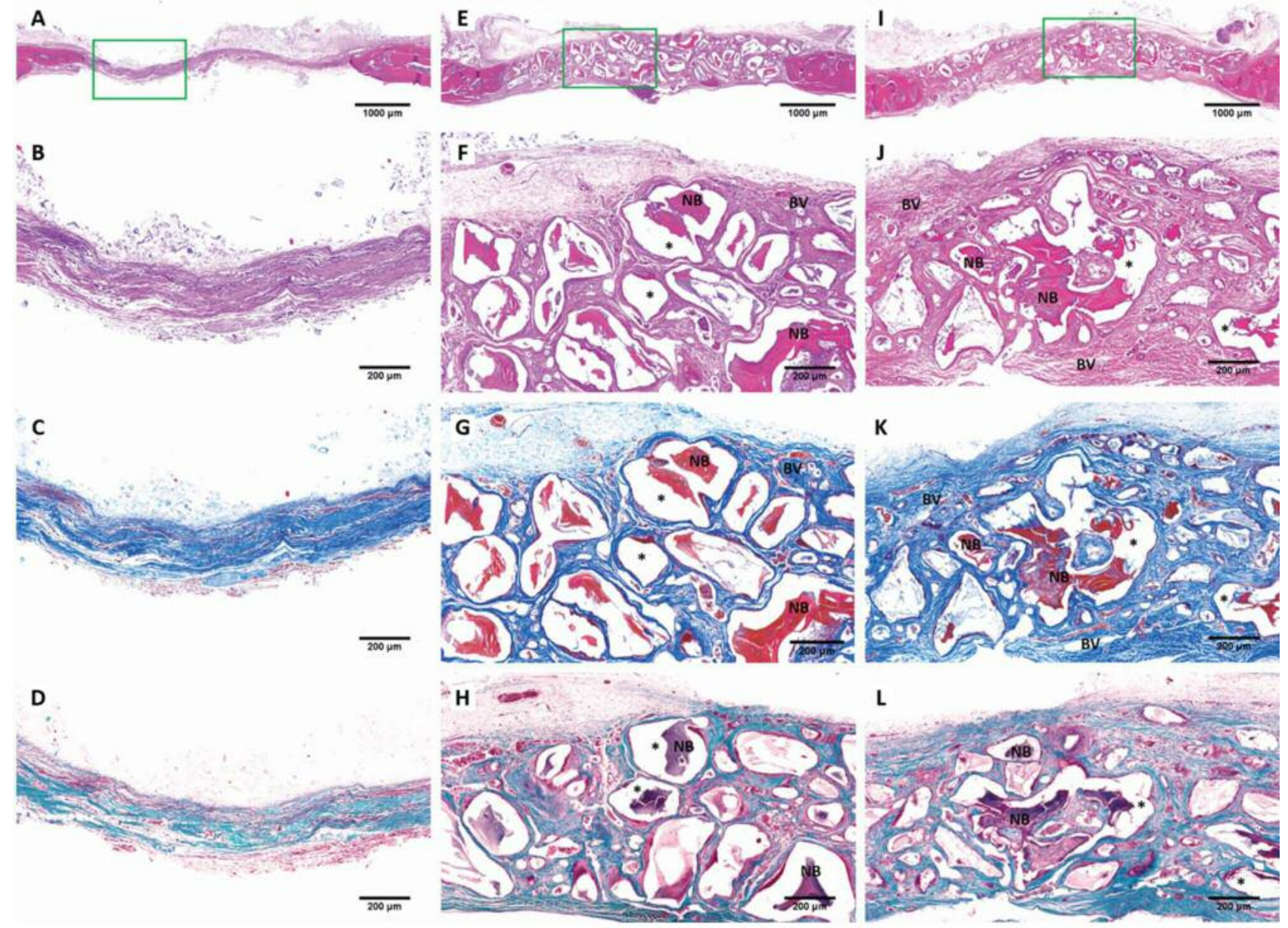

Figure 7. Histological findings of rat calvarial defects at 4 weeks after implantation $(A-D)$ the $C D$ group, $(E-H)$ the $B C$ group, and $(I-L)$ the PHBB group. Samples $(A, B, E, F, I$, and $J)$ were stained with $H \& E$. Samples $(C, G$, and $K)$ were stained with Masson's Trichrome. Samples $(D, H$, and $L)$ were stained with Goldner's Trichrome. *Residual grafts, BV: blood vessel, NB: newly formed bone. [(A, E, I: original magnification $\times 40)$, (B, C, $D, F, G, H, J, K, L$ : original magnification $\times 200)]$.

developed the bone substitute (PHBB), we used a rat criticalsized calvarial defect model and compared the characteristics of PHBB with those of BC.

Examination of the surface structures of BC and PHBB by LV-SEM revealed irregularly and fully interconnecting macropores. These macropores generally enhance resorption and facilitate the movement of $\mathrm{Ca}$ and $\mathrm{P}$ ions into the intercellular medium beyond the bone graft material, improving osteoconductivity (10-12). Thus, both BC and PHBB were expected to show good osteoconductivity in vivo.

The chemical properties of the BC and the PHBB were assessed using ATR-FTIR spectroscopy. The FTIR spectra of porcine cancellous bone, one of the constituents of the PHBB, showed the characteristic peaks of PO43- (1,020 and $\left.600-550 \mathrm{~cm}^{-1}\right)$ and carbonate $\left(1,454\right.$ and $\left.1,444 \mathrm{~cm}^{-1}\right)$, which are typical hydroxyapatite peaks (13). Porcine type I collagen, another constituent of PHBB, showed five typical bands of collagen at the frequencies of 3,296, 2,920, 1,647, 1,544 , and $1,236 \mathrm{~cm}^{-1}$ corresponding to amide A, B, I, II, and III, respectively (14). The spectrum of PHBB was characterized by absorption bands arising from hydroxyapatite $\left(1,444,1,020\right.$, and $\left.600-550 \mathrm{~cm}^{-1}\right)$ and collagen $\left(1,680\right.$ and $\left.1,647 \mathrm{~cm}^{-1}\right)$.

The compressive strength of BC decreased dramatically from $53.49 \pm 11.59 \mathrm{~N}$ to $4.86 \pm 0.88 \mathrm{~N}$ when it was wet. In comparison, the difference in compressive strength between dry and wet PHBB was smaller than that of BC $(11.22 \pm 1.53$ vs. $2.33 \pm 0.37 \mathrm{~N}$, respectively). PHBB exhibited approximately one half of the compressive strength of $\mathrm{BC}$ under wet condition. However, the compressive strength of dental bone graft is not a very critical factor once the bone graft can maintain the space and act well as a bone void filler. 

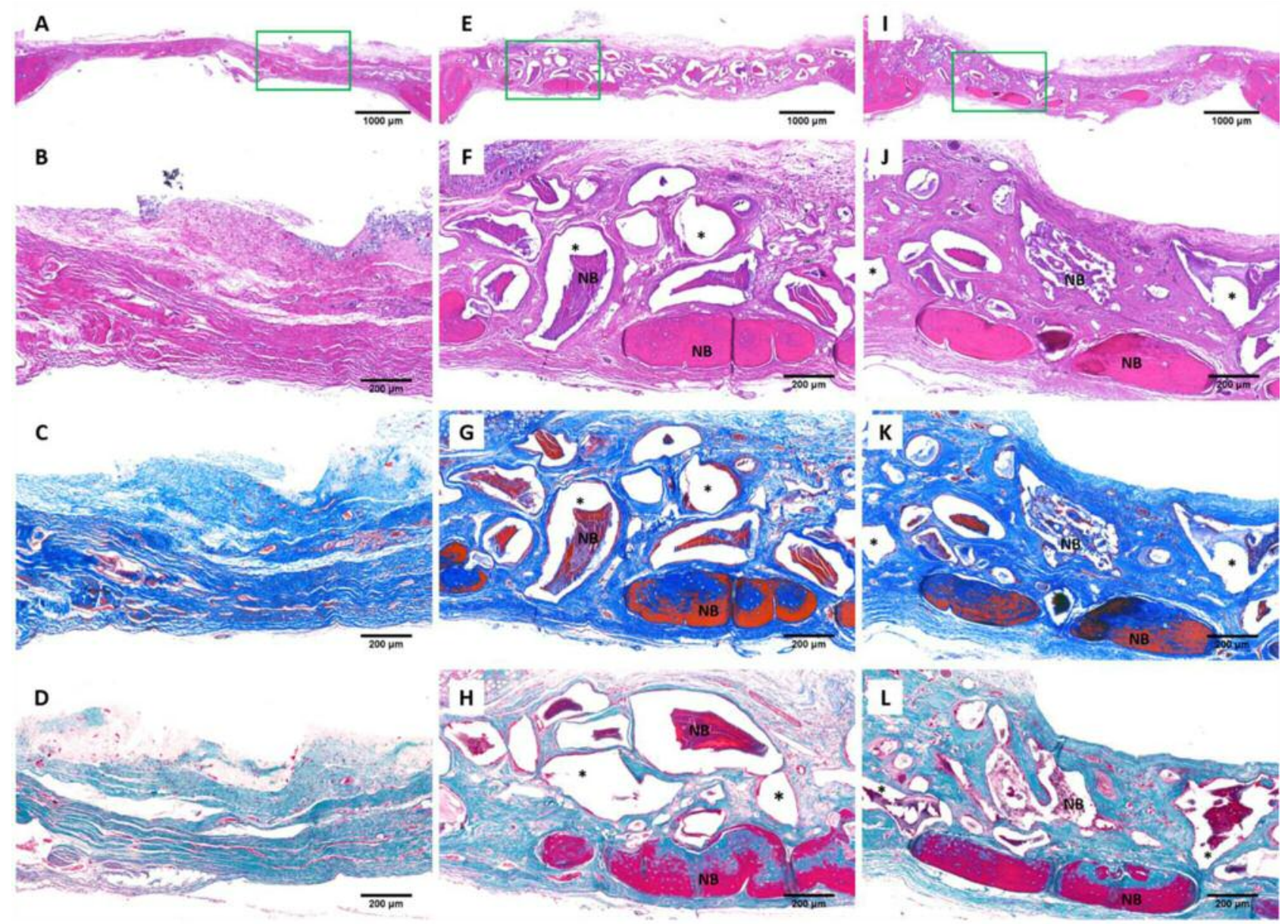

Figure 8. Histological findings of rat calvarial defects at 8 weeks after implantation $(A-D)$ the CD group, (E-H) the BC group, and (I-L) the PHBB group. Samples $(A, B, E, F, I$, and $J)$ were stained with $H \& E$. Samples $(C, G$, and $K)$ were stained with Masson's Trichrome. Samples $(D, H$, and $L)$ were stained with Goldner's Trichrome. *Residual grafts, BV: blood vessel, NB: newly-formed bone. [(A, E, I: original magnification X40), (B, C, $D, F, G, H, J, K$, L: original magnification $\times 200)]$.

The flexibility of PHBB was assessed by checking resilience of the wet sample after the removal of compression. PHBB showed good recovery to its original shape when the compression was removed. This property can be attributed to type I collagen that holds hydroxyapatite granules together and enhances handling characteristics of PHBB.

In the animal study, well-localized materials at the grafted site were confirmed by micro- $\mathrm{CT}$ analysis. Both BC and PHBB groups showed significant differences in bone mineral density and bone volume compared to the $\mathrm{CD}$ group at 4 and 8 weeks after bone graft. And interestingly, the BC-PHBB ratio of $\mathrm{BMD}$ and $\mathrm{BV}$ were decreased over time after implantation indicating that new bone formation occurs faster in the PHBB group than in the BC group.

In histological analysis, the defect site of the $\mathrm{CD}$ group was collapsed with a small amount of connective tissue. In contrast, the defect sites of the BC and PHBB groups were well maintained with residual grafts, bone-like material, and newly-formed bone in addition to fibrous connective tissue. At week 4, the PHBB group showed more angiogenesis than the BC group. This higher angiogenesis may cause mesenchymal cells to differentiate into osteoblast progenitors, particularly during intramembranous ossification (15). Moreover, blood vessels in the bone graft site supply various growth factors including vitamins and parathyroid hormones (PTHs) which facilitate the healing process, particularly at an early stage of bone healing (16). At week 8 , replacement of the grafted materials with bony tissue was prominent in the PHBB group. This indicates that the bone particles of PHBB degrade faster than the Bio-OSS ${ }^{\circledR}$ granules, providing more space for new bone formation. These results are consistent with those of other studies describing the fast resorption rate 
of porcine bone grafts $(16,17)$. Typically, faster resorption of grafted materials is expected to induce earlier substitution with new bone (15), and thus a similar result was predicted for the PHBB grafted site (18).

\section{Acknowledgements}

This research was supported by a grant of the Next-Generation BioGreen 21 Program (No.: PJ01135201), Rural Development Administration and Korea Institute of Planning and Evaluation for Technology in Food, Agriculture, Forestry and Fisheries (IPET) through Agri-Bio Industry Technology Development Program, funded by Ministry of Agriculture, Food and Rural Affairs (MAFRA) (No. 316031-3).

\section{References}

1 Kato E, Lemler J, Sakurai K and Yamada M: Biodegradation property of beta-tricalcium phosphate-collagen composite in accordance with bone formation: a comparative study with BioOss Collagen ${ }^{\circledR}$ in a rat critical-size defect model. Clin Implant Dent Relat Res 16(2): 202-211, 2014.

2 Jung UW, Lee JS, Park WY, Cha JK, Hwang JW, Park JC, Kim CS, Cho KS, Chai JK and Choi SH: Periodontal regenerative effect of a bovine hydroxyapatite/collagen block in one-wall intrabony defects in dogs: a histometric analysis. J Periodontal Implant Sci 41(6): 285-292, 2011.

3 Murata M: Collagen biology for bone regenerative surgery. J Korean Assoc Oral Maxillofac Surg 38(6): 321-325, 2012.

4 Isobe Y, Kosaka T, Kuwahara G, Mikami H, Saku T and Kodama S: Oriented collagen scaffolds for tissue engineering. Materials 5(3): 501-511, 2012.

5 Dong $\mathrm{C}$ and Lv Y: Application of collagen scaffold in tissue engineering: recent advances and new perspectives. Polymers $8(2): 42,2016$.

6 InTech: Advances in composite materials for medicine and nanotechnology https://www.intechopen.com/books/advances-incomposite-materials-for-medicine-and-nanotechnology/advancesin-collagen-hydroxyapatite-composite-materials (2011, Published online 01 April 2011).

7 Gleeson JP, Plunkett NA and O'Brien FJ: Addition of hydroxyapatite improves stiffness, interconnectivity and osteogenic potential of a highly porous collagen-based scaffold for bone tissue regeneration. Eur Cell Mater 20: 218-230, 2010.

8 Kim Y, Nowzari H and Rich SK: Risk of prion disease transmission through bovine-derived bone substitutes: a systematic review. Clin Implant Dent Relat Res 15(5): 645-653, 2013.

9 Go A, Kim SE, Shim KM, Lee SM, Choi SH, Son JS and Kang SS: Osteogenic effect of low-temperature-heated porcine bone particles in a rat calvarial defect model. J Biomed Mater Res A 102(10): 3609-3617, 2014.
10 Cyster LA, Grant DM, Howdle SM, Rose FR, Irvine DJ, Freeman D, Scotchford CA and Shakesheff KM: The influence of dispersant concentration on the pore morphology of hydroxyapatite ceramics for bone tissue engineering. Biomaterials 26(7): 697-702, 2005.

11 Mehdizadeh H, Sumo S, Bayrak ES, Brey EM and Cinar A: Three-dimensional modeling of angiogenesis in porous biomaterial scaffolds. Biomaterials 34(12): 2875-2887, 2013.

12 Ramírez-Fernández MP, Calvo-Guirado JL, Maté-Sánchez del Val JE, Delgado-Ruiz RA, Negri B and Barona-Dorado C: Ultrastructural study by backscattered electron imaging and elemental microanalysis of bone-to-biomaterial interface and mineral degradation of porcine xenografts used in maxillary sinus floor elevation. Clin Oral Implants Res 24(5): 523-530, 2013.

13 Sionkowska A and Kozłowska J: Characterization of collagen/hydroxyapatite composite sponges as a potential bone substitute. Int J Biol Macromol 47(4): 483-487, 2010.

14 Al-Munajjed AA, Plunkett NA, Gleeson JP, Weber T, Jungreuthmayer C, Levingstone T, Hammer $\mathrm{J}$ and O'Brien FJ: Development of a biomimetic collagen-hydroxyapatite scaffold for bone tissue engineering using a SBF immersion technique. J Biomed Mater Res B Appl Biomater 90(2): 584-591, 2009.

15 Jensen SS, Broggini N, Hjørting-Hansen E, Schenk R and Buser $\mathrm{D}$ : Bone healing and graft resorption of autograft, anorganic bovine bone and $\beta$-tricalcium phosphate. A histologic and histomorphometric study in the mandibles of minipigs. Clin Oral Implants Res 17(3): 237-243, 2006.

16 Kanczler JM and Oreffo RO: Osteogenesis and angiogenesis: the potential for engineering bone. Eur Cell Mater 15(2): 100-114, 2008.

17 Ramírez-Fernández M, Calvo-Guirado JL, Delgado-Ruiz RA, Maté-Sánchez del Val JE, Vicente-Ortega V and MeseguerOlmos L: Bone response to hydroxyapatites with open porosity of animal origin (porcine [OsteoBiol ${ }^{\circledR} \mathrm{mp} 3$ ] and bovine [Endobon ${ }^{\circledR}$ ]): a radiological and histomorphometric study. Clin Oral Implants Res 22(7): 767-773, 2011.

18 Scarano A, Degidi M, Iezzi G, Pecora G, Piattelli M, Orsini G, Caputi S, Perrotti V, Mangano C and Piattelli A: Maxillary sinus augmentation with different biomaterials: a comparative histologic and histomorphometric study in man. Implant Dent 15(2): 197-207, 2006.
Received August 14, 2018

Revised September 18, 2018

Accepted September 24, 2018 